\title{
Hautprobleme durch schlechte Büroluft?
}

\author{
Haut- und Augenprobleme am Arbeitsplatz führen Büroangestellte \\ gern auf die schlechte Luftqualität zurück. Eine deutsche Studie \\ stellte diese These jetzt auf den Prüfstand.
}

m Rahmen des ProKlimA-Projektes war von 1994-1999 die physikalische, chemische und biologische Belastung von Räumen in insgesamt 14 deutschen Bürobauten ermittelt und in Relation zu subjektiven und objektiven medizinischen Befunden gesetzt worden. Erfurter Arbeitsmediziner benutzten jetzt diese Daten und fokussierten in einer Subanalyse auf die Hautprobleme der Teilnehmer: Bei 925 Büroangestellten waren mit dem Sebumeter der Lipidgehalt und mit dem Korneometer die Hydratation der Haut bestimmt worden. In einem Fragebogen hatten die Teilnehmer über persönliche Hautprobleme und das Ausmaß der subjektiven Belästigung berichtet.

Die Wahrscheinlichkeit für ein subjektiv berichtetes Hautproblem korre- lierte in den Regressionsanalysen positiv mit der Zugehörigkeit zum weiblichen Geschlecht (Odds Ratio [OR] 2,3), schlechter Software bei der Computerarbeit (OR 2,2), negativen Arbeitsplatzcharakteristika (OR 1,8), allergischen Erkrankungen (OR 1,5) und dem Gebrauch von Hautcremes (OR 2,6).

In der anschließenden objektiven medizinischen Analyse ergab sich eine signifikante Korrelation von geringer Hautfeuchtigkeit und dem Gehalt an flüchtigen organischen Verbindungen wie Hexen und Hexadekan in der Luft (OR 2,5) sowie einer geringen Luftfeuchtigkeit im Raum (OR 1,9). Der Hautlipidgehalt war dagegen unabhängig von diesen beiden Luftqualitätsparametern.

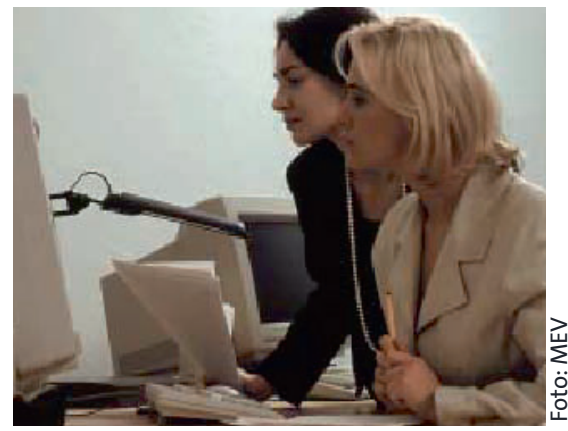

Schlechtes Betriebsklima setzt der Haut offenbar mehr zu als schlechtes Raumklima.

Fazit: Klagen Büroangestellte über trockene, rissige oder gerötete Haut, so ist dies eher auf arbeitsplatzbezogene Faktoren sowie das Geschlecht als auf die Innenraumluft zurückzuführen. $\quad b k$

Brasche S et al. Comparison of risk factor profiles concerning self-reported skin complaints and objectively determined skin symptoms in German office workers. Indoor Air 2004; 14: 137-43

tung gut oder sehr gut, während die Plazebobehandlung überwiegend eine mäßige bis schlechte Bewertung erhielt (89\% bzw. 87\%).

Die Verträglichkeit war gut, relativ sicher auf Vitamin B12 zurückzuführende Nebenwirkungen waren Brennen oder Jucken (zwei Patienten) sowie Rötung (ein Patient) und Überwärmung mit „Ameisenlaufen“ (ein Patient). Die Nebenwirkungen verschwanden im Laufe weniger Tage. Die durch Vitamin B12 hervorgerufene Rotfärbung der Haut kann zunächst als störend empfunden werden, wird aber dann allgemein akzeptiert.

Fazit: Die topische Applikation von Vitamin B12 ist offenbar eine effektive und gut verträgliche Behandlungsoption bei Patienten mit atopischer Dermatitis. $b k$

Stücker $\mathbf{M}$ et al. Topical vitamin B12 a new therapeutic approach in atopic dermatitis - evaluation of efficacy and tolerability in a randomized placebocontrolled multicentre clinical trial. Br J Dermatol 2004; 159: 977-83 